\title{
2
}

\section{Information Technology Assimiliation in Schools: A Theoretical and Practical Framework}

\author{
Moshe Telem ${ }^{a}$ and Ben-Zion Barta ${ }^{b}$ \\ ${ }^{a}$ School of Education, Tel Aviv University, Tel Aviv, Israel \\ ${ }^{b}$ Ministry of Education, Culture and Sports, Jerusalem, Israel
}

\begin{abstract}
Information Technology (IT) assimilation constitutes a big challenge for schools. An IT assimilation framework (ITAF) in schools is suggested. The main elements in each of its components are presented and the framework's potential is indicated as a tool for effective and successful implementation, operation and usage of IT in schools. An integrated example and guidelines for school employees' preparation programs conclude our discussion.
\end{abstract}

Keyword Codes: J.1; K.6.1; H.4.2

Keywords: Administrative Data Processing, Education; Management of Computing and Information Systems, Project and people Management; Information Systems Applications, MIS.

\section{INTRODUCTION}

To our best knowledge, the issue of IT assimilation into school administration has not received, so far, a thorough and focused discussion in the literature. The absence of such a discussion is particularly felt due to the rapid computerization process strived after by and taking place in schools. School management information systems (MISs) are being designed and implemented [1] to provide educational administrators with new tools to support them in a variety of activities like: grade and attendance reporting; placement in classes; teachers' allocation to classes; construction of school time-tables and examination schedules; assignment and disbursement of resources; follow-up on the implementation of decisions; analysis of teacher and school achievements, etc. At the same time, automated office tools (e.g., electronic mail, electronic archives, spreadsheets, automatic follow-up of decisions, electronic appointment books, automatic dialing, desk-top publishing) are rapidly becoming routine. The school, as a small organization and lacking IT professionals is not well prepared to cope with the technological change [2].

IT's introduction into the traditional school structure has run into difficulties [3,4] and even dangers [5]. Situations where computers installed into traditional school structures are not fully and effectively used are frequently reported [6]. As a result IT's use and effectiveness are limited, and administrators are frustrated [7, p. 284]. 
The same is true for school districts. A survey showed that the number of management applications of IT, and the dependency upon it for conduct of business affairs in school districts are on the increase, but it was also found "(a) that most districts have too much, 'data' and too little 'information', (b) that requests for information outstrip the capacities of existing systems, and (c) that requests for data or information often fall outside existing categories or filing systems" [ 8 p.198]. Though these findings come from the end of the 80's, the professional literature does not seem to indicate significant changes during the recent years.

The absence of an overall, integrated approach for IT assimilation in schools is, very often, a dominant factor in this failure, resulting also in (or perhaps from ?) the faulty perception that IT introduction is merely the purchase and installation of hardware and software, accompanied by some introductory training. Rejecting this perception, this paper suggests an IT assimilation framework (ITAF) for schools. It is reasonable to assume that ITAF will significantly contribute to a more successful IT introduction and usage in schools and educational authorities.

\section{THE IT ASSIMILATION FRAMEWORK}

\subsection{Theoretical foundations}

The incorporation of IT into the day-to-day activity of schools is not merely a technical-oriented issue, but rather has a strong and overall impact on the entire school structure and dynamics. ITAF should, therefore, reflect this point of view. Current systems approach, which is also a "new and emerging approach in educational administration as from the 1970s" [9, p. 128], provides the theoretical foundations for ITAF. The systems approach typifies Kast and Rosenzweig's [10] view of organizations. According to them the school, like any organization, is composed of five major, partly overlapping subsystems: managerial, structural, psychosocial, goals and values, and technical. Introducing a change in one of them would lead to changes in the other four. These five subsystems provide the foundation for ITAF. Ahead we shall present an overall, integrated approach for IT assimilation in schools based on these theoretical foundations.

Due to the complexity and variety of elements in each of the five ITAF components, the examples under each of the components do not attempt to encompass all ITAF issues.

\subsection{ITAF subsystems}

\subsubsection{The managerial subsystem}

As in any organization, the main elements of the school's managerial subsystem are: goal setting, planning, organizing, assembling resources, staffing, directing, coordinating, budgeting, evaluating, implementing and controlling $[10, \mathrm{p} .115]$. As classified in the above theoretical foundations, each of these elements is atiected by 11 introduction, and assimilation activities should relate to what implications IT introduction would have on each of them and to the training needed to cope with them. For example: school's computerization policy formulation; specifying information requirements; IT assimilation master-plan preparation; IT introduction and performance evaluation; re-definition of resources allocation; staffing - recruiting of IT professionals or changing existing roles for this purpose; redefining school's computerized control (and supervision) procedures (i.e. looser versus tighter school coupling; consideration of "quality control" regulation in schools), etc. 


\subsubsection{The structural subsystem}

IT introduction may result in the addition of new roles in the school (e.g. the school computer administrator) as well as in far-reaching implications on school's work flow, authority, information flow, procedures and rules. Within the school environment assimilation activities should therefore relate to issues such as changes in the school's formal organizational structure; necessary changes in the information flow within the school and between the school and its external environment; effects on interrelations between school employees; etc.

\subsubsection{The psychosocial subsystem}

The main elements of the school's psychosocial subsystem are: human resources, attitudes, perceptions, motivation, group dynamics, leadership, communication and interpersonal relations [10, p. 114-15]. IT assimilation results in the school's need to confront a cluster of psychosocial issues associated with IT assimilation and aggravated by computer illiteracy of the school's instructional and administrative staff [11].

Examples of IT effects on the components of this subsystem's could be the need to identify potential barriers to IT introduction into school, or the development of standards for professional development programs for school administrators and teachers (see paragraph 4). Changes in relationships between school employees (teacher-teacher, teacher-counselor, counselor-principal, etc.) should be, if possible, forecasted and frequently investigated. Appropriate steps, whenever necessary, to introduce changes in communication patterns within the school and between the school and its external environment, should be taken.

\subsubsection{The goals and values subsystem}

The main elements of the school's goals and values subsystem are: culture, philosophy, overall goals, group goals and individual goals [10, p.114]. Each of these elements is effected by IT introduction. Examples of required activities within this subsystem are the formulation of a new "school philosophy" for the computerized school; the effort to define IT introduction goals for administrators and teachers; the detection of changes occurring in the school's interrelations with its environment; ethical use of IT for educational purposes; dealing with legal aspects of IT (e.g. security and data confidentiality in school), and using information provided by IT to define school goals in a less ambiguous way.

\subsubsection{The technical subsystem}

The school's technical subsystem is shaped by the following elements: knowledge, techniques, facilities and equipment [10, p.114]. Each of these elements should be adapted selectively to suit the special requirements of the school, and to make appropriate use of technology - technology "as a process and not as a product" [12]. Examples of appropriate activities within this subsystem are the preparation of computer sites; purchase, introduction, operation and maintenance of hardware and software; adaptation of new knowledge areas and techniques necessary for this purpose. 


\section{AN EXAMPLE}

In order to illustrate an integrated, overall IT assimilation process, the introduction of a management information system (MIS) into schools will serve as an example. Due to the variety of elements, the complexity and scope of each of the five ITAF subsystems, the following is just a partial example that does not attempt to cover all ITAF issues.

The technical subsystem is significantly changed: Information requirements (defined by the managerial subsystem) have to be translated into IT specifications, e.g. development or purchase of MIS software, choice and purchase of suitable hardware, communications, operating system and office automation tools and their installation in a (sometimes new) computer site and on employees' desks all over the school.

The structural subsystem is immediately affected by the changes in the technical subsystem. The addition of a new role - that of the school computer administrator - will result in a change in the school's formal organizational structure. The establishment of a central integrated database replacing the manual archives and filing cabinets will result in changes in existing roles and information flow. For example, the computer on the principal's/counselor's/etc. desk, gives him or her, direct access to the school's integrated database, thus minimizing his or her dependency on information middlemen. The information retrieved is more updated and broader than before IT introduction. New statistical and comparative analyses are available.

The psychosocial subsystem is affected as well. Some school employees might resist the change while others may be enthusiastic, with a potential of conflict between the two groups. Recruitment of new staff, necessitating IT-oriented qualifications for new and/or existing roles might be necessary. School employees development programs should be carried out (see paragraph 4).

The goals and values subsystem undergoes change as well. Administrators and teachers have to understand that computers do not transform students into numbers and that IT in an additional administrative (and instructional) tool for achieving school administrative and instructional goals, for example: identification of students needing special educational care, excelling/underachieving students, and so on. Criteria on ethical use of information and for data security and confidentially, should be formulated and strictly enforced on all school employees.

IT introduction into the managerial system provides school employees with information and tools hitherto unavailable. These may assist them in decision making, management and follow up in their areas of responsibility, as mentioned under 2.2.1. The actual changes occurring in school management should be thoroughly researched. The principal should initiate, manage and supervise the above mentioned changes in the four other subsystems. $\mathrm{S} / \mathrm{He}$ and other school employees should use MIS as an aggressive educational leadership tool [5, 13], enabling them to perform their various administrative and pedagogical tasks. For this purpose, the principal and other school employees should acquire (through adequate preparation programs) new knowledge areas relating to MIS assimilation, operation and usage. Equipped with this new knowledge the principal should be responsible for a precise definition of the major objectives of MIS introduction for administrative and instructional purposes. S/He should formulate policy guidelines and an effective school MIS strategy within a master-plan for MIS introduction, including a clear definition of the principal's information needs and those of all of the school employees. 
The principal should control MIS introduction and be capable of managing school's MIS. The principal (and other school employees) should be cognizant that MIS, as a managerial tool, is a means and not an end in itself, and that it should primarily serve the pedagogical needs of school.

\section{SCHOOL EMPLOYEE PREPARATION}

Proper school employee preparation programs are a pre-requisite for IT's successful assimilation. ITAF should become an integral part of the curriculum of universities and other institutions preparing school administrators, so that the latter will learn to understand ITAF's objectives, advantages, limitations and its day to day operation; IT's possible applications; IT's potential uses as an aggressive educational leadership tool and as a pro-active management tool; IT's potential for school administration (and instruction) and as leadership enhancing professional productivity tool. Principals should also be trained to prepare contingency frameworks for IT assimilation in their specific school situations, and to understand the organizational contexts for a successful IT assimilation.

Specific preparation programs should be prepared for:

a) principals and school management staff.

b) school computer administrators.

c) administrative staff.

d) teaching staff.

e) assistance staff (e.g. counselors, psychologists).

Training activity should take place prior to IT implementation and subsequently on an on-going basis familiarize staff with hardware and software changes and recent developments. Proper preparation is a pre-requisite for overcoming school employees possible resistance to change (see paragraph 3).

\section{CONCLUSION}

The computerized school in which administrators and teachers will be working in the late 1990's will be far different from today's school. One of the main conclusions of this paper is that IT introduction affects the entire spectrum of school activities in its five main separate yet interrelating subsystems: the managerial, structural, psychosocial, goals and values and technical. The ITAF suggested in this paper matches each of these subsystems. A master plan for an efficient, reliable and controlled IT assimilation in school is very much recommended. ITAF is vital for successful IT assimilation in school administration: Without it, very often, IT is introduced in ignorance which either results in failure, or even worse, creates the danger of transforming school into an "industrial" organization. It is hoped that this paper will raise awareness and recognition of the importance and potential of ITAF for IT assimilation in schools. 


\section{REFERENCES}

1. Journal of Research on Computing in Education, 24(1), 1991.

2. M. Telem, Managing information growth and implementation in small organizations. Information Processing and Management, 24(5), (1989), 443-452.

3. A. Fainmesser and S. Mendel, Integrating schools in educational information systems: Pilot project conclusions. (Hebrew). Jerusalem, The Israeli Ministry of Education, Culture and Sports, 1989.

4. M. Mackett, F. Frank and P. Abrams, Educational information systems: A resource for education. The Journal of Information Resources Management 1(2), (1988), 2-28.

5. M. Telem, DSS in educational organizations. Computers and Education, 14(1), (1990), 61-69.

6. H. Beare, B.J. Caldwell and R.H. Millikan, Creating an excellent school: Some new management techniques. London, Routledge, 1989.

7. J.W. Guthrie, W.I. Garms and L.C. Pierce, School finances and education policy enhancing educational efficiency, equality and choice. Englewood Cliffs, NJ, Prentice-Hall, 1988.

8. L.L. Cunningham, Computers and educational governance. In J.A. Culberston and L.L. Cunningham (eds.). Microcomputers and education, eighty-fifth Yearbook of the National Society for the Study of Education, pp. 193-281. Chicago, University of Chicago, 1986.

9. W.R. Scott, Organization. Englewood Cliffs, NJ, Prentice-Hall, 1981.

10. F.E. Kast and J.E. Rosenzweig, Organization and management: A systems and contingency approach. Tokyo, McGraw-Hill, 1985.

11. M. Telem, Conceptual and operational considerations for the planning and implementation of a pedagogical MIS on a national scale. Programmed Learning and Educational Technology, 24, (1987), 187-193.

12. W.C. Bozemann, Technology and the design of decision support systems in education. UCEA Review, 20, (1979), 3-8.

13. M. Telem, Educational DSS: Potential services, benefits, difficulties and dangers. Computers and Education, 14(1), (1990), 70-81. 